\title{
A Clinico Epidemiological Study of Periocular Hyperpigmentation
}

\author{
Neeraja Sobha Kartha1, Balachandran Parapattu Kunjukunju² \\ ${ }^{1}$ Department of Dermatology, Venereology and Leprosy, Government T.D. Medical College, Alappuzha, Kerala, India. \\ ${ }^{2}$ Department of Dermatology, Venereology and Leprosy, Government T.D. Medical College, Alappuzha, Kerala, India.
}

\section{ABSTRACT}

\section{BACKGROUND}

Periorbital pigmentation is a relatively common condition encountered in dermatology practice. The aetiology of periocular pigmentation may be multifactorial. There is a scarcity of data regarding the prevalence of the condition mainly because of its transitory and floating nature, the lack of reasonable etiologic explanations, and the fact that the condition is considered just a cosmetic nuisance. We wanted to study the clinical and epidemiological characteristics of periocular hyperpigmentation and assess the proportion of various determinants in adult patients.

\section{METHODS}

A descriptive study was done among 130 adult patients with periocular pigmentation who attended a tertiary care hospital in Kerala during a period of one and a half years. A detailed history, dermatological and system examination, Wood's lamp \& relevant laboratory investigations were done and results were analysed using appropriate biostatistics.

\section{RESULTS}

Majority of the patients belonged to $2^{\text {nd }}$ decade and females predominated. Atopy and regular cosmetic usage were seen in $72 \%$ of the patients. Involvement of both eyelids was seen in $53.8 \%$ \& post inflammatory was the most common type seen. Iron deficiency anaemia was the commonly associated systemic illness seen and stress aggravated the condition in $55.4 \%$ of our patients. $51.5 \%$ had grade 3 pigmentation. Wood's lamp examination showed a predominance of epidermal pigmentation in $49.2 \%$ patients.

\section{CONCLUSIONS}

Although considered a phenomenon within the limit of physiology, patients, especially women, are really bothered and concerned about it, even relating the presence of dark circles with significant impairment on their quality of life. A proper understanding of the etiopathogenesis is important which includes identification and therapeutic targeting of each contributing etiologic factor for an individual patient.

\section{KEY WORDS}

Periocular Pigmentation, Multifactorial, Wood's Lamp, Individualized Treatments
Corresponding Author:

Dr. Balachandran Parapattu Kunjukunju, Professor,

Department of DVL,

Government T.D. Medical College,

Alappuzha-688005, Kerala, India.

E-mail: balu.parapattu@gmail.com

DOI: 10.14260/jemds/2020/149

Financial or Other Competing Interests: None.

How to Cite This Article:

Kartha NS, Kunjukunju BP. A clinico epidemiological study of periocular hyperpigmentation. J. Evolution Med. Dent. Sci. 2020;9(09):687-691, DOI: 10.14260/jemds/2020/149

Submission 27-12-2019,

Peer Review 04-02-2020,

Acceptance 11-02-2020,

Published 02-03-2020.

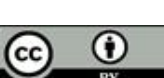




\section{BACKGROUND}

Periocular pigmentation is an extremely common yet unexplored condition in Indian patients. It is known by various names like periorbital melanosis, dark circles, infraorbital pigmentation or Idiopathic Cutaneous Hyperchromia of Orbital Region (ICHOR). It is defined as bilateral, round, homogenous pigmented macules on the periocular region, worsened by generalized fatigue especially lack of sleep. The condition is more pronounced in certain ethnic groups and are also frequently seen in many members of the same family. The condition interferes with facial appearance, giving the patient a tired, sad or hangover look. ${ }^{1}$ Despite the high prevalence of this condition, there are few published studies regarding the aetio pathogenesis. ${ }^{1}$ The earliest references to this condition was by Touraine et al in 1937 who said that dark circles may be due to prominence of vessels due to loss of subcutaneous fat and muscle tonus in the region. ${ }^{2}$ The condition occurs in both sexes with an increasing frequency in females, owing to the hormonal factors and seen more frequently in dark skinned patients, especially Asians. $(3,4)$

The condition worsens with the aging process of skin sagging and altered subcutaneous fat distribution. ${ }^{5}$ While there are not much statistics giving the frequency of its occurrence, judging from the sheer number of products advertised to either lighten or cover the pigmentation, dark circles are a cosmetic concern for large number of individuals. ${ }^{6}$ Periorbital pigmentation can broadly be divided into primary and secondary types.

Primary Type- It is also known as Idiopathic Cutaneous Hyperchromia of the Orbital Region (ICHOR), characterized by bilateral darkening of the orbital skin and eyelid, which is not secondary to systemic or local diseases. ${ }^{7}$

Secondary Type- It has a multifactorial pathogenesis including genetic or constitutional, excessive pigmentation due to dermal melanin deposition, post inflammatory hyperpigmentation secondary to atopic and allergic contact dermatitis, periorbital oedema, excessive subcutaneous vascularity, shadowing due to skin laxity and tear trough associated with aging. ${ }^{8}$

The degree of pigmentation varies with certain factors such as fatigue, menstrual cycle and other situations of physical and mental stress, owing to dermal vessel clogging during such periods. ${ }^{9}$

\section{Clinical Types of Periorbital Pigmentation 10}

A. Constitutional- It is seen as a curved band of brownish to black pigmentation on the skin of the lower eyelids approximating the shape of the orbital rim with frequent involvement of upper eyelids.

B. Post inflammatory- It is characterized by the presence of irregular patches of brownish or grey pigmentation on the skin on the upper, lower or both eyelids with features of lichenification, accentuation of skin creases, and eczematous papules or patches in the surrounding areas. It can occur secondary to atopic or allergic contact dermatitis due to cosmetics.
C. Vascular- This type is characterized by the presence of erythema predominantly involving the inner aspect of lower eyelids, with prominent capillaries or telangiectasia or the presence of bluish discoloration of the lower eyelid and veins that becomes more prominent when the overlying skin is stretched. This type of dark circle appears to be due to a combination of transparency of the overlying skin and dermal vascularity.

D. Shadow Effect- Presence of a dark shadow under an overhanging tarsal muscle, eye bags, or the presence of a deep tear trough over the medial aspect of inferior orbital rim that disappear with direct lighting.

E. Others - Other causes including anaemia, Nevus of ota, hormonal disturbances, nutritional deficiencies, acanthosis nigricans, associated chronic illness, habits, stress etc. 11

A clinical approach to the treatment of periocular pigmentation should include identification and therapeutic targeting of each contributing etiologic factor for an individual patient. Treatment options include bleaching creams, topical retinoic acid, chemical peels, laser therapies, injectable fillers, fat transfer and surgery, used either as monotherapy or in combination. ${ }^{1}$ If there is pigment deposition, depigmenting agents are the most suitable drugs. If the cause is skin laxity the treatment is mainly procedural, with lasers \& surgery. If the cause is translucent eyelids and visible vasculature, autologous fat transplantation or soft tissue fillers can be used. ${ }^{1}$ There are only limited number of Indian studies regarding periocular pigmentation, despite the common occurrence of this condition. Hence this study attains importance in the present settings.

\section{METHODS}

A descriptive study was done on 130 adult patients in the age group of 18-60 years attending a tertiary care hospital in Kerala, during a period of 18 months, after getting an informed consent. Aim and Objectives were to study the Clinical \& Epidemiological characteristics and to assess the proportion of various determinants of Periocular pigmentation. All the patients who presented with active dermatitis in the periocular area were excluded from the study. Name, age, gender, history of atopy, cosmetic use, family history, site of lesion, Clinical types, concurrent systemic illnesses and associated factors were recorded in the pre-structured proforma.

A detailed dermatologic examination was done in all patients including grading of the pigmentation, clinical tests (eyelid stretch test, Gaze \& Direct lighting) and Wood's lamp examination.

Grading of $\mathrm{POH}$ in Comparison to Surrounding Skin 12

0 - Skin colour comparable to other facial skin areas

1 - Faint pigmentation of infraorbital fold

2 - Pigmentation more pronounced

3 - Deep dark colour, all lids involved

4 - Grade 3+ pigmentation spreading beyond infraorbital fold. 


\section{Clinical Tests for Periorbital Pigmentation ${ }^{8}$}

1. Eyelid Stretch Test- The test is done by stretching the lower eyelid. If the dark circles improve, then it is due to skin laxity. If dark circles worsen, it is due to subdermal vascularity. If no change in dark circles, then it is due to pigment deposition.

2. Gaze Test- The test differentiates fat from fluid (oedema) over the periocular area. Periorbital fat disappears in downgaze and appears in upgaze. Compared with normal orbital fat, oedema is still present in upgaze \& downgaze.

3. Wood's Lamp- Wood's lamp is used to differentiate between epidermal and dermal pigmentation. If epidermal, there is accentuation of the pigment with UV rays while there is no change for dermal pigmentation.

Relevant investigations like complete hemogram, thyroid profile, Random blood sugar, serum cholesterol, liver function tests and renal parameters were done. The data was properly coded and entered in Microsoft Excel and analysed using SPSS software. Appropriate tests of significance were applied.

\section{RESULTS}

A total of 130 patients with periocular melanosis were included in the study during a period of 18 months. Most of the patients $(36.9 \%)$ belonged to the age group of $21-30$ years. The mean age of the study population was 35.08 years. The youngest patient was 19 years old and the oldest was 57 years old. (Figure-1)

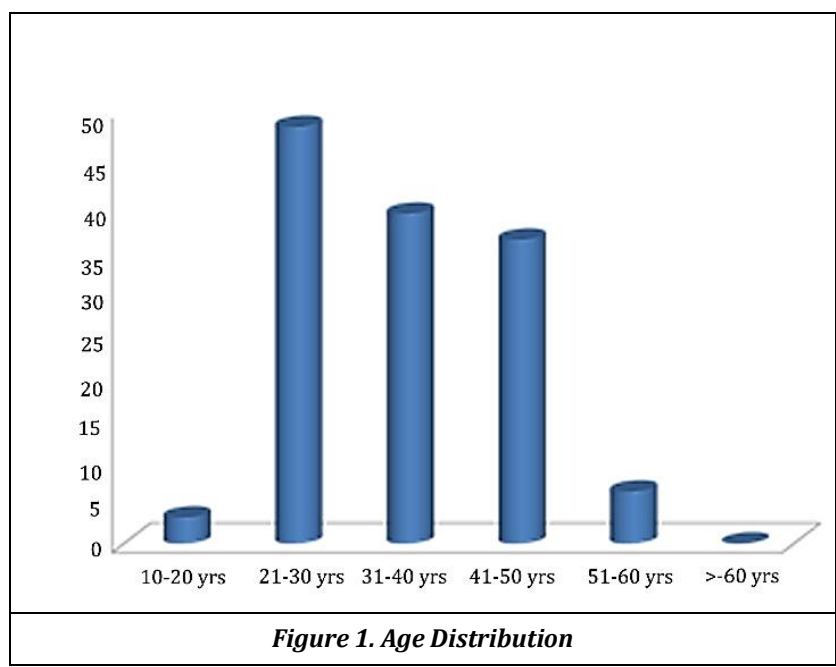

Out of 130 patients studied, 31 (24\%) were males and 99 (76\%) were females. In the present study, 94 patients (72\%) had history of atopy \& 95 (73\%) gives history of regular use of cosmetics. Among our 130 patients, 70 (53.8\%) had pigmentation over both upper and lower eyelids while 55 (42.3\%) had pigmentation over the lower eyelid. Only 1 patient $(0.8 \%)$ had pigmentation limited to upper eyelid and $4(3.1 \%)$ had pigmentation over other areas also. The most common type observed was Post inflammatory (40\%), followed by Skin laxity (13.1\%), Vascular (12.3\%) \& tear trough $(10 \%)$. The least observed were combinations of the above types. (Table -1). Among our 130 patients, Iron deficiency anaemia was seen in $61(46.9 \%)$ patients followed by thyroid disorders in 19 patients (14.6\%). Least common was renal disorder (3.1\%). 16 patients $(12.3 \%)$ had no concurrent systemic illnesses. (Table -2 ). Stress was seen in $72(55.4 \%)$ of our patients which was followed by faulty habits in 17 (13.1\%) There were no associated factors in 29 $(22.3 \%)$ of patients. (Table -3 ).

\begin{tabular}{|c|c|c|}
\hline Types & No. of Patients & $\mathbf{\%}$ \\
\hline Constitutional & 19 & 14.6 \\
\hline Post Inflammatory & 52 & 40.0 \\
\hline Vascular & 16 & 12.3 \\
\hline Nevoid & 2 & 1.5 \\
\hline Periorbital edema & 6 & 4.6 \\
\hline Skin laxity & 17 & 13.1 \\
\hline Deep tear trough & 13 & 10.0 \\
\hline Combination of Vascular, Skin laxity \& Tear trough & 3 & 2.3 \\
\hline Combination of Vascular \& Skin laxity & 2 & 1.5 \\
\hline Total & $\mathbf{1 3 0}$ & $\mathbf{1 0 0}$ \\
\hline Table 1. Types of Periocular Pigmentation \\
\hline
\end{tabular}

\begin{tabular}{|c|c|c|}
\hline Systemic Illness & No. of Patients & Percentage \\
\hline Iron deficiency anaemia & 61 & 46.9 \\
\hline Thyroid disorders & 19 & 14.6 \\
\hline Presence of both above disorders & 15 & 11.5 \\
\hline Liver disorder & 10 & 7.7 \\
\hline Hormonal imbalances & 5 & 3.8 \\
\hline Renal disorder & 4 & 3.1 \\
\hline No systemic illness & 16 & 12.3 \\
\hline Total Table 2. Systemic IIIness & $\mathbf{1 0 0}$ \\
\hline \multicolumn{2}{|c}{} \\
\hline
\end{tabular}

\begin{tabular}{|c|c|c|}
\hline Associated Factors & No. of Patients & Percentage \\
\hline Stress & 72 & 55.4 \\
\hline Faulty habits & 17 & 13.1 \\
\hline Drugs & 6 & 4.6 \\
\hline Uncorrected errors of refraction & 6 & 4.6 \\
\hline No associated factors & 29 & 22.3 \\
\hline Total Table 3. Associated Factors \\
\hline \multicolumn{2}{|c}{} \\
\hline
\end{tabular}

Among our 130 patients, majority ( 51.5\%) had Grade 3 pigmentation followed by grade 4 in $30.8 \%$ and Grade 2 in $16.9 \%$ of patients. The least observed was grade $1(0.8 \%)$. (Figure-2) On performing the clinical tests, 18 (13.8\%) patients showed improvement with the eyelid stretch test, 18 $(13.8 \%)$ showed worsening while $94(72.3 \%)$ patients had no change. Only $7(5.4 \%)$ had positive gaze test and disappearance of pigmentation with direct lighting was seen in $36(27.7 \%)$ of patients. Out of 130 patients, epidermal pigment deposition was seen in 64 (49.2\%) patients while only $3(2.3 \%)$ showed dermal pigment deposition.

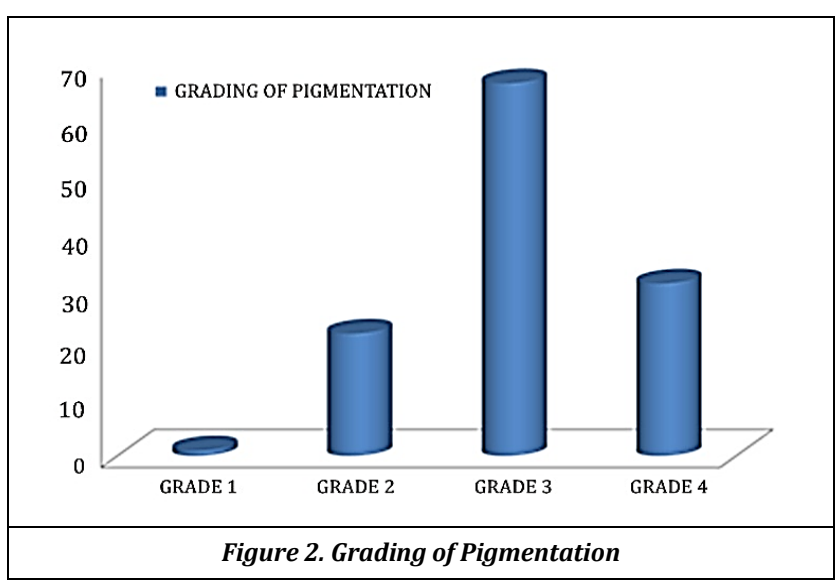




\section{DISCUSSION}

Periocular pigmentation is an extremely common yet unexplored condition in Indian patients. The condition is more pronounced in certain ethnic groups and are also frequently seen in many members of the same family. ${ }^{1}$ In the present study, majority of patients $(36.9 \%)$ belonged to the age group of 21-30 years. This is in concordance with the study done by Sheith and Shah et al where majority of the patients fell into 16-25 years. ${ }^{12}$ This may be due to the fact that people in this age group are more concerned about their outward appearance and who are regular cosmetic users.

Our study included 31 males and 99 females with male to female ratio being $1: 3.2$, which is in concordance with study done by Sheith and Shah et al who also reported a ratio of 1:4.2.12 The above finding is in contrast to study done on western population by Yaar et al who reported a ratio of $1: 1 .{ }^{5}$ This contradictory result may be due to the racial differences and varied triggering factors in our population when compared to western world, on whom majority of studies have been done. In our study, 94 (72.3\%) patients had history of atopy. This is in contrast to the study done by Sheith et al who reported atopy in only $33 \%$ of patients and Ranu et al who reported atopy in $55 \%$ patients. ${ }^{(10,12)}$ This may be one of the reasons for the predominance of Post inflammatory type of periocular pigmentation we obtained in our study.

In the present study, majority of patients, 95 (73\%) regularly used cosmetics which is in concordance with the study by Shah et al, suggesting that there is some ingredient in cosmetics which can cause allergic contact dermatitis and leave behind post inflammatory pigmentation. ${ }^{12}$ In our study, both upper and lower eyelids were equally affected (53.8\%) which is in contrast to study by Sheith et al where lower eyelid was involved in majority (72.5\%). ${ }^{12}$ Our study revealed only one patient who had pigmentation confined to upper eyelid alone, which is in contrast to results obtained by Sarma et al who found upper eyelid pigmentation to be much more common in occurrence. ${ }^{13}$ The above observations may be due to the genetic variations among different populations in the architecture of the periocular region.

In our study, $52(40 \%)$ patients had Post inflammatory type followed by Constitutional in 19 (14.6\%) patients, Skin laxity in 17 (13.1\%), Vascular in $16(12.3 \%)$ patients and Deep tear trough in $13(10 \%)$ patients. The least obtained type was combination of the above factors. The increased frequency of Post Inflammatory type is probably due to increased incidence of atopy and use of cosmetics seen in our population. Our findings were in contrast to the few Indian studies done by Sheith et al and Ranu et al who reported Vascular \& Constitutional as the common types respectively.(10,12) Shadow effect is due to presence of a dark shadow created by an overhanging tarsal muscle or eyebags, which is due to periorbital oedema/ fat, skin laxity or deep tear trough that disappear with direct lighting. In our study, periocular pigmentation due to shadow effect was observed in $36(27.7 \%)$ patients. This is much higher when compared to the study by Shah et al who reported shadowing only in $11.4 \%$ patients. ${ }^{12}$ The reduced number of patients showing combination of the above types were in consistent with other
Indian and Western studies. 2 patients with unilateral blue grey pigmentation due to Nevus of Ota was obtained.

Iron Deficiency anaemia was the most common systemic illness seen associated with periocular pigmentation in our study, seen in 61 (46.9\%) patients, followed by thyroid disorders in 19 (14.6\%) and combination of both. Among thyroid disorders, Hypothyroidism was seen in all patients. Periocular pigmentation due to liver disorder was seen in 10 (7.7\%), 5 (3.8\%) was having hormonal disturbances (3 were having PCOS and 2 were taking OCPs), 4 (3.1\%) had renal disorder and $16(12.3 \%)$ had no associated systemic illnesses. The increased incidence of Iron deficiency anaemia was in concordance with the study done by Sheith et al who reported the deficiency in $50 \%$ patients. This may be either because enough oxygen is not reaching the periorbital tissues or due to facial pallor which makes the periorbital region look comparatively darker. ${ }^{12}$ Many patients reported improvement of dark circles on correction of anaemia. Gendler et al stated that some medical problems that may contribute to dark circles include disorders of thyroid, kidney or liver, metabolic and endocrine disorders. However, statistical data was not shown in this literature.

Stress, both occupational \& non-occupational, was seen aggravating the periocular pigmentation in 72 (55.4\%) of patients, which is in concordance with the results obtained by Sheith et al who obtained stress in $71 \%$ patients. ${ }^{12}$ Stress induced exacerbation may be due to effect of increased MSH secretion via HPA axis in response to stress which creates a vicious cycle. According to Gathers et al, fatigue, stress \& emotional liability all may play a significant role in development of periocular pigmentation. ${ }^{14}$ Other associated factors included faulty habits such as frequent eye rubbing and lack of adequate sleep was seen in 17 (13.1\%) patients, drug intake was seen with $6(4.6 \%)$ patients. Another 6 $(4.6 \%)$ patients had uncorrected errors of refraction while 29 $(22.3 \%)$ patients had no associated factors.

The above findings are somewhat lower than the results obtained by Sheith and Shah et al who reported lack of adequate sleep in $40 \%$, frequent eye rubbing in $32.50 \%$ and lack of correction for errors of refraction like myopia in $12 \%$ patients. ${ }^{12}$ According to Gathers et al, faulty habits cause significant strain on eyelid muscle, leading to their exhaustion which can aggravate periocular pigmentation. ${ }^{14}$ In our study, majority of the patients $(51.5 \%)$ had grade 3 pigmentation, involving all the eyelids followed by grade 4 pigmentation in $31 \%$ and grade 2 pigmentation in $16.9 \%$. The least seen was grade 1 pigmentation $(0.8 \%)$. This is in contrast to results obtained by Shah et al who obtained grade 2 pigmentation in $57.5 \%$ patients. ${ }^{12}$ The predominance of darker pigmentation seen in our study may be due to post inflammatory pigmentation over periocular area secondary to atopic or allergic contact dermatitis and darker skin types.

Eyelid stretch test showed improvement in 18 (13.8\%) patients and showed worsening in $18(13.8 \%)$ patients. Improvement with eyelid stretch test indicate shadow effect, either due to skin laxity or deep tear trough. Worsening on stretching the lower eyelid indicates visible vasculature due to overlying thin skin. Gaze test is used to differentiate between periorbital fat and fluid. If there is no change with upgaze and downgaze, it indicates presence of periorbital fluid. Disappearance on downgaze and appearance on upgaze 
indicates periorbital fat. In our study, 7 (5.4\%) patients had periorbital fat while none had periorbital oedema as the cause of periocular pigmentation. Direct lighting is used to identify periocular pigmentation due to shadow effect. In our study, $36(27.7 \%)$ patients had disappearance of their pigmentation on direct lighting. Wood's lamp is used mainly to differentiate between epidermal and dermal melanin deposition. Epidermal pigmentation shows accentuation while there is no change with dermal melanin deposition. In our study, 64 (49.2\%) patients had epidermal pigmentation while only $3(2.3 \%)$ had dermal pigmentation. Out of these 3 , 2 presented with Nevus of Ota. This is in contrast to earlier studies where majority of patients had dermal pigmentation. ${ }^{12}$

\section{CONCLUSIONS}

Periocular pigmentation is an extremely common yet unexplored condition in Indian patients. The most common age group affected was found to be $21-30$ years and M:F ratio was $1: 3.2$. Among our patients, $72.3 \%$ of our patients had a history of atopy and $73 \%$ of our patients were regular cosmetic users. The site most commonly affected was both upper and lower eyelids (53.8\%). The most common type was post inflammatory type $(40 \%)$ followed by constitutional type (14.6\%). Iron deficiency anaemia was the common systemic illness associated in $46.9 \%$ patients. Stress was seen to be aggravating the condition in $55.4 \%$ of our patients. $51.5 \%$ of our patients showed grade 3 pigmentation. Wood's lamp examination showed predominance of epidermal pigmentation in $49.2 \%$. Despite being a common problem, treatment to an individual patient is not always given. So, a proper understanding of aetiopathogenesis and good clinical examination can render a better successful outcome for the patient.

\section{REFERENCES}

[1] Freitag FM, Cestari TF. What causes dark circles under the eyes? J Cosmet Dermatol 2007; 6 (3):211-5.

[2] Touraine A. Dermatoses: symptomatic visceral lesions. Surgical Medical Encyclopedia, Dermatology 1937; 2:14.
[3] Gendler EC. Treatment of periorbital hyperpigmentation. Aesthet Surg J 2005; 25 (6):618-24.

[4] Sarkar R. Idiopathic cutaneous hyperchromia at the orbital region or periorbital hyperpigmentation. J Cutan Aesthet Surg 2012; 5 (3):183-4.

[5] Yaar M, Gilchrest BA. Skin aging: postulated mechanisms and consequent changes in structure and function. Clin Geriatr Med 2001; 17 (4):617-30.

[6] Alsaad SM, Mikhail M. Periocular hyperpigmentation: a review of etiology and current treatment options. J Drugs Dermatol 2013; 12 (2):154-7.

[7] Verschoore M, Gupta S, Sharma VK, et al. Determination of melanin and haemoglobin in the skin of Idiopathic Cutaneous Hyperchromia of the Orbital Region (ICHOR): a study of Indian patients. J Cutan Aesthet Surg 2012; 5 (3):176-82.

[8] Chatterjee M. Periocular pigmentation. In: Lahiri K, Chatterjee M, Sarkar R, eds. Pigmentary disorders: a comprehensive compendium. $1^{\text {st }}$ edn. Jaypee Publishing 2014: p. 366-75.

[9] Roh MR, Chung KY. Infraorbital dark circles: definition, causes and treatment options. Dermatol Surg 2009; 35(8):1163-71.

[10] Ranu H, Thng S, Goh BK, et al. Periorbital hyperpigmentation in Asians: an epidemiologic study and a proposed classification. Dermatol Surg 2011; 37(9):1297-303.

[11] Lowe NJ, Weider JM, Shorr N, et al. Infraorbital pigmented skin. Preliminary observations of laser therapy. Dermatol Surg 1995; 21(9):767-70.

[12] Sheth PB, Shah HA, Dave JN. Periorbital hyperpigmentation: a study of its prevalence, common causative factors and its association with personal habits and other disorders. Indian J Dermatol 2014; 59(2):1517.

[13] Sarma N, Chakraborty S, Bhattacharya SR. Acquired, Idiopathic, Patterned Facial Pigmentation (AIPFP) including periorbital pigmentation and pigmentary demarcation lines on face follows the lines of blaschko on face. Indian J Dermatol 2014; 59(1):41-8.

[14] Gathers RC. Periorbital hypermelanosis. In: Paul KA, edr. Dermatologyfor skin of color. $1^{\text {st }}$ edn. New York: McGraw- Hill 2009: p. 341-3. 